\title{
Sensing the Material Position in Construction Project
}

\author{
NIE Wen-hui , ZOU Zhi-wen, JU Shi-guang \\ School of Computer Science \& Telecommunication Engineering, Jiangsu University, zhenjiang, Jiangsu, \\ 212013,China \\ whnie@ujs.edu.cn,zzw_yj@126.com,jushg@ujs.edu.cn
}

\begin{abstract}
In order to improve the monitoring and controlling performances in construction projects, especially in multi-story projects, This paper researched the approaches to track the materials and components on construction project. A locating model in 3 dimensional spaces is proposed. The mentioned locating algorithms are compared, and shortages and advantages of each are given. The purpose is to find an optimal solution to manage the location information in construction project site with lower costs, higher locating accuracy and ideal reliability.
\end{abstract}

Index Terms - construction project, material management, 3D positioning, wireless sensor networks

\section{Introduction}

Of all the factors to raise the project management levels in construction projects, materials management is an important fact which plays an important role .Studies have showed that a very basic materials plan and approach would produce a minimum $6 \%$ savings in craft labor $\operatorname{cost}^{[1][2]}$, and that an additional 4 to $6 \%$ savings would probably be made by integrated computer-aided

Manuscript received January 15, 2009; revised June 21, 2009; accepted July 28, 2009.

Supported by nature science foundation of China(no.60773049)、 Jiangsu project innovation for PHD candidates(CX07B_125z) and zhenjiang social development foundation(SH2008028). Zhenjiang Scientific Project(GY2008030) systems that track bulk materials line items. This additional savings to the ability of the crafts is related to schedule their work around material availability. Management on labor productivity, reporting ineffective use of work-hours equivalent to $18 \%$ and loss in labor productivity.

Triangulation, scene analysis, and proximity are the three main principal techniques for automatic location sensing. Location systems usually employ them individually or in combination ${ }^{[5]}$.

Location based services (LBS) require a reliable, accurate and continuous position determination of mobile objects. This is particularly true in relatively enclosed environment, such as a construction project site, where the widely used Global Positioning System (GPS) is not available due to its signal outages. To solve this problem, one solution is to integrate different techniques in a multi-sensor positioning system to overcome the limitations of a single sensor. In this paper an approach is proposed using a three-dimensional Radio Frequency Identification (3D RFID) location algorithm, (3D-based Positioning Model and Algorithm in Ad Hoc ,3D-PMWSN) to locate the nodes in WSN. In this model, grid in space is presented. When one tag is detected by a certain reader whose position is known, that tag's position can be known through certain

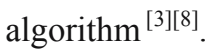

For the great importance of construction material position management, recent researches have researched the feasibility of follows: (1) automatically tracking the location of construction agents (laborer and equipment), (2) identifying and determining the status of the basic activity that the agent is engaged in, and (3) deriving 
project performance indicators ${ }^{[2]}$. However, most researches have mainly focused on 2 dimensional environment and construction agents, not fully examined the potential of tracking the location of materials on a construction site and 3 dimensional positioning. This paper discusses such issues.

\section{Localization algorithm}

Most localization techniques are based on the received radio strength indicator (RSSI) due to its widely availability in wireless radio signal communication ${ }^{[4]}$. Specially, RSSI-based localization has an advantage to use the same radio hardware for both communication and localization; this would make it possible to provide efficiency in simple design framework over a specific localization infrastructure - such as ones using directional antenna or same transmission signal, and separate design of ultrasound or infrared ${ }^{[3]}$.

\section{$2.1 \quad$ ZigBee approach}

ZigBee is emerging network technology and a wireless communication standard capable of realizing the ubiquitous environment to satisfy such requirements as a superset of IEEE 802.15.4 standard ${ }^{[4]}$, ZigBee supports the industrial network standards, so that many industrial applications, including construction automation, structural health monitoring and automated control, can benefit from the advantages of the technology. ZigBee specification takes advantage of the IEEE 802.15.4 wireless protocols as communications method, and expands on this with a flexible mesh network, wide range of applications and interoperability. The ZigBee specification has been released publicly in June 2005, and products supporting the ZigBee standard are widely available in the market nowadays.

A new localization technique with combination of radio frequency and ultrasound was presented for more accurate positioning performance. A feasibility analysis showed that combination of radio frequency and ultrasound will provide a better performance in measurement accuracy than the one that uses only $\mathrm{RF}^{[4]}$. Using different channel allocation supported by ZigBee standards, various sensor-based devices can be embedded into this system capable of providing versatile monitoring capacities for different purposes. Investigation indicated that automated field data acquisition in construction site can benefit from the development of this system with respect to the future collaboration mechanism with several project management platforms.

\subsection{Fuzzy logic algorithm}

Fuzzy logic systems include three important kinds of neural networks defined, i.e. linear neural networks, rectangle wave neural networks and nonlinear neural networks. It is proved that nonlinear neural networks can be represented by rectangle wave neural networks.

The fuzzy logic method used in tracking object builds on the insights gained through the Dempster-Shafer approach $^{[4]}$; it could consider the model to be continuous in some control variables such as moving tags or readers which are discreticized in the other algorithms described earlier. This conceptual method is under development. It is being encoded and the new field experiments began in 2006.

\subsection{Triangulation based on transmission space}

The triangulation location sensing technique uses the geometric properties of triangles to compute object locations ${ }^{[5]}$. Triangulation is divisible into the subcategories of late ration, using distance measurements, and angulations using primarily angle or bearing measurements.( see Figure 1.)

In Figure $1, \mathrm{O}_{1}, \mathrm{O}_{2}$ and $\mathrm{O}_{3}$ are three non-collinear points whose positions are known before. Radar or other equipment which can send signals is set in each point. $\mathrm{P}$ denotes the object to be positioned. If $\mathrm{P}$ is detected by readers in $\mathrm{O}_{1}, \mathrm{O}_{2}$ and $\mathrm{O}_{3}$, for the angles of $\gamma \mathrm{i}, \beta_{\mathrm{i}}$ and $\alpha_{i}(i=1,2)$ can already be known, so the position of $p$ can be easily calculated . 


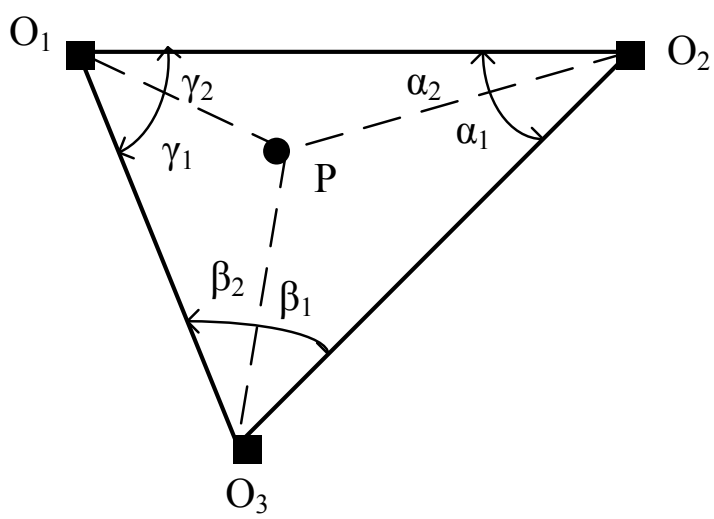

Figure 1. Locating a position unknown object using Triangulation approach

There are three general approaches to measure the distances: i.e. Direct, Time-of-Flight and Attenuation. Let's discuss this:

(1) Direct measurement uses a physical action or movement. This method is simple, but the distance is relatively difficult to obtain.

(2) Time-of-Flight approach calculates the distances between an object and some referenced points by the signal travel time ${ }^{[6]}$. The famous application is GPS(Global Positioning System). The principle behind GPS is the measurement of distance (or "range") between the satellites and the receiver. The satellites tell us exactly where they are in their orbits by broadcasting data the receiver uses to compute their positions. It works something like this: if we know our exact distance from a satellite in space, we can know we are somewhere on the surface of an imaginary sphere with a radius equal to the distance to the satellite radius. If we know our exact distance from two satellites, we know that we are located somewhere on the line where the two spheres intersect. And, if we take a third and a fourth measurement from two more satellites, we can find our location. The GPS receiver processes the satellite range measurements and produces its position.

(3) A proximity location sensing technique entails determining when an object is "near" a known location (see figure 2). The object's presence is sensed using a physical phenomenon with limited range. There are three general approaches to sensing proximity ${ }^{[5]}$. The object is in the intersection of $r_{1}, r 2$ and $r_{3}$.In other words, the object is near $r_{1}, r_{2}$ and $r_{3}$.

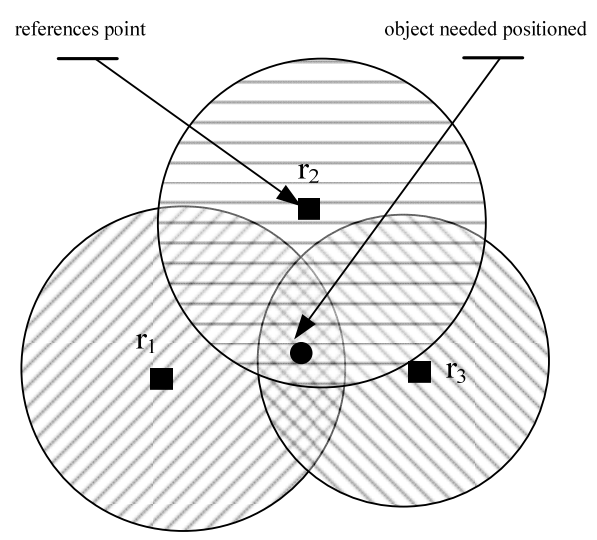

Figure. 2 proximity location sensing technique

\subsection{A 3 dimension-based positioning method 3D-PMAHN approach}

\subsubsection{D-PMAHN model introduction}

Firstly, a cube Q space region is set up, which is called operation region in space (see Figure. 3 ), then we divide each side of $\mathrm{Q}$ into $\mathrm{n}$ same parts. Accordingly, the cube $\mathrm{Q}$ is divided into $\mathrm{n}^{3}$ small cube called space cells. For each cell, it has an exclusive cell coordinate $(\mathrm{x}, \mathrm{y}, \mathrm{z}),(1<=\mathrm{x}<=\mathrm{n}, 1<=\mathrm{y}<=\mathrm{n}, 1<=\mathrm{z}<=\mathrm{n}) \quad$.Here we define $n^{3}$ be Q's positioning resolution. Obviously, when $\mathrm{Q}$ is fixed, the bigger $\mathrm{n}$ is, the bigger the positioning resolution rate will be ${ }^{[7][8]}$.

Then, we scatter randomly $\mathrm{m}$ readers whose positions are known in $\mathrm{Q}$ and assume that each reader is equipped with a same RF transceiver. Every reader has a sphere communication region with radius $r$. The needed positioning object is a tag in Q. So far as the tag lies in any readers' communication regions, it can be captured by these readers. In other words, the readers can read the tag in its communication region. Our purpose is to locate a tag in $\mathrm{Q}$ by readers whose positions are known .

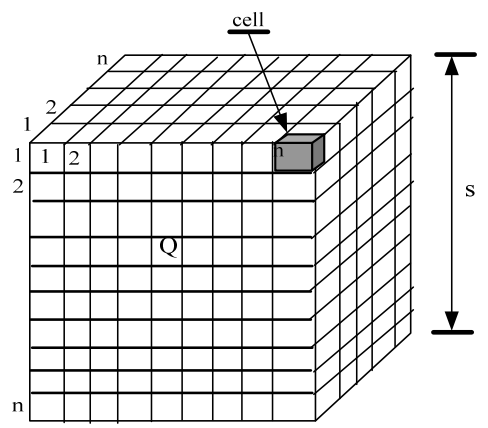

Figure. 3 3D-PMAHN Model 
In order to simplify calculation, we assume that (1)The operation region is fixed.(2)The number of cells is variable.(3) All readers in $Q$ have the same communication regions G. (4) Parameter $r$ is variable. The main approach for the localization algorithm can be generally described as follows: firstly, scattering some necessary readers with known positions in $\mathbf{Q}$. The size of a cell can be gained through calculation. A reader's communication region $\mathbf{S}$ can be showed by the number of cells contained in it. Once a tag lies in two readers' communication regions, we can deduce that the tag's position must be in the intersection of this two readers' communication regions, and the size and position of this intersection can be gained by simple calculation .Similarly, if one tag can be captured by more than two readers, the tag's position can be gained by the intersection of these readers' communication regions.

\subsubsection{D-PMAHN Localization Algorithm}

let's analyze the reader communication region 's features in 3D space (see Figure. 4)

Assume that region is a sphere. In case of parameter $n$, $r$ and $s$ are fixed, letting the side be 2 plus $x$ for the max inner cube ${ }^{[7]}$.

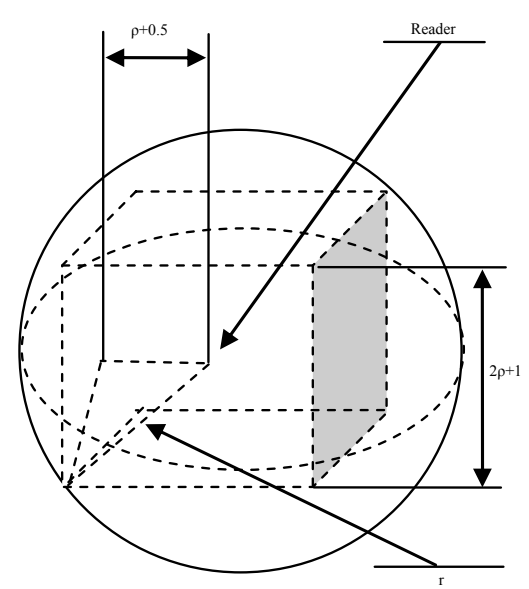

Figure 4. Reader's communication region $\mathbf{G}$ in 3D-PMWSN

Obviously,

$$
\begin{aligned}
& 3 \mathrm{x}^{2}=\mathrm{r}^{2}, \text { then } \\
& \rho=\left[\frac{n r}{\sqrt{3} s}\right]
\end{aligned}
$$

where $[x]$ is the integer part of $x$, the unit of $\rho$ is the number of cell.
Accordingly, node S can communicate with every other node in the cube centered at $\mathrm{S}$ and containing $(2 \rho+1)^{3}$ cells.

Assuming that tag $T_{i}$ can be captured by readers $R_{1}, R_{2} \ldots$ $\mathrm{R}_{\mathrm{k}}$ at the same time, then it is obvious that this tag is in the communication regions' intersection of above readers.[7]

Let

$T=Q \cap \bigcap_{i=1}^{k} S_{i}$

$\left.\left.\left.=Q \cap\left[x_{+}-\rho, x_{-}+\rho\right)\right] \times\left[y_{+}-\rho, y_{-}+\rho\right)\right] \times\left[z_{+}-\rho, z_{-}+\rho\right)\right](4)$

Where

$$
\begin{array}{ll}
\mathrm{x}_{+}=\max \left(\mathrm{x}_{1}, \ldots, \mathrm{x}_{\mathrm{m}}\right), & \mathrm{x}_{-}=\min \left(\mathrm{x}_{1}, \ldots, \mathrm{x}_{\mathrm{m}}\right) \\
\mathrm{y}_{+}=\max \left(\mathrm{y}_{1}, \ldots, \mathrm{y}_{\mathrm{m}}\right), & \mathrm{y}_{-}=\min \left(\mathrm{y}_{1}, \ldots, \mathrm{y}_{\mathrm{m}}\right) \\
\mathrm{z}_{+}=\max \left(\mathrm{z}_{1}, \ldots, \mathrm{z}_{\mathrm{m}}\right), & \mathrm{z}_{-}=\min \left(\mathrm{z}_{1}, \ldots, \mathrm{z}_{\mathrm{m}}\right)
\end{array}
$$

where $\max \left(x_{m}, x_{n}\right)$ is the bigger one of $x_{m}$ and $\mathrm{x}_{\mathrm{n}}, \min \left(\mathrm{x}_{\mathrm{m}}, \mathrm{x}_{\mathrm{n}}\right)$ is the smaller one of $\mathrm{x}_{\mathrm{m}}$ and $\mathrm{x}_{\mathrm{n}}$, $\max \left(\mathrm{y}_{\mathrm{m}}, \mathrm{y}_{\mathrm{n}}\right), \min \left(\mathrm{y}_{\mathrm{m}}, \mathrm{y}_{\mathrm{n}}\right), \max \left(\mathrm{z}_{\mathrm{m}}, \mathrm{z}_{\mathrm{n}}\right)$ and $\min \left(\mathrm{z}_{\mathrm{m}}, \mathrm{z}_{\mathrm{n}}\right)$ have the similar meanings.

The simulation result shows that the 3D-PMAHN is simple and energy saving. Such characters meet the special requirements in Ad Hoc networks ${ }^{[7]}$.

\subsection{Qualitative comparison of above localization models}

\section{(1) Localization dimension}

Seen from the latest report, ZigBee approach overcome the limitations of previous RFID-based and GPS-based technologies in current construction material positioning practices, a new ZigBee localization technique with combination of radio frequency and ultrasound was presented for more accurate positioning performance[4],nonetheless, it is still in 2 dimensional space. Fuzzy logic algorithm and Triangulation based on transmission algorithm are relatively proposed earlier than ZigBee approach, can locate an object mainly in a two dimensional areas. Only 3D-PMAHN can works in 3 dimensional spaces.

\section{(2) Algorithm cost}

The system cost is always one of the most concerned 
factors, especially for application oriented research. Because of the low price of a single ZigBee node, the ZigBee algorithms has the lowest $\operatorname{cost}^{[10]}$.Through the realization in Ad Hoc networks, it has been showed that 3D-PMAHN has medium $\operatorname{cost}^{[8]}$.The other approaches are logical models, so their application costs relies on their practical realization strategies.

\section{(3) Positioning accuracy}

Accuracy is one of the most important requirements for resources tracking at construction sites ${ }^{[10]}$. Such accuracy seems to be difficult to achieve based on RSSI (received signal strength indicator) at construction sites in light of the highly nonlinear fashion of radio signal propagation and severe multipath fading or shadowing effects. In addition, unstable and fast-changing surroundings at building sites may further degrade the performance of RSSI localization. Therefore, accurate models of signal propagation are expected to be established by extensive site tests and using nonlinear statistical models. The positioning accuracy of ZigBee approach is relatively high, Fuzzy logic algorithm is medium, Triangulation based on transmission is high, 3D-PMAHN in medium.

\section{(4) Feasibility}

Apart from 3D-PMAHN, every algorithm has been successfully used in application .However, emulation has showed that 3D-PMAHN is feasible ${ }^{[8][10]}$.

In short, 3D-PMAHN is a relatively new positioning approach with the property of lower cost, higher positioning accuracy. Specially, it can works in 3 dimensional spaces. This is a suitable approach to use in material tracing on construction site and we have used it in application of material management.

\section{Application in construction project}

The positioning procedure is as follows(references to figure 5)

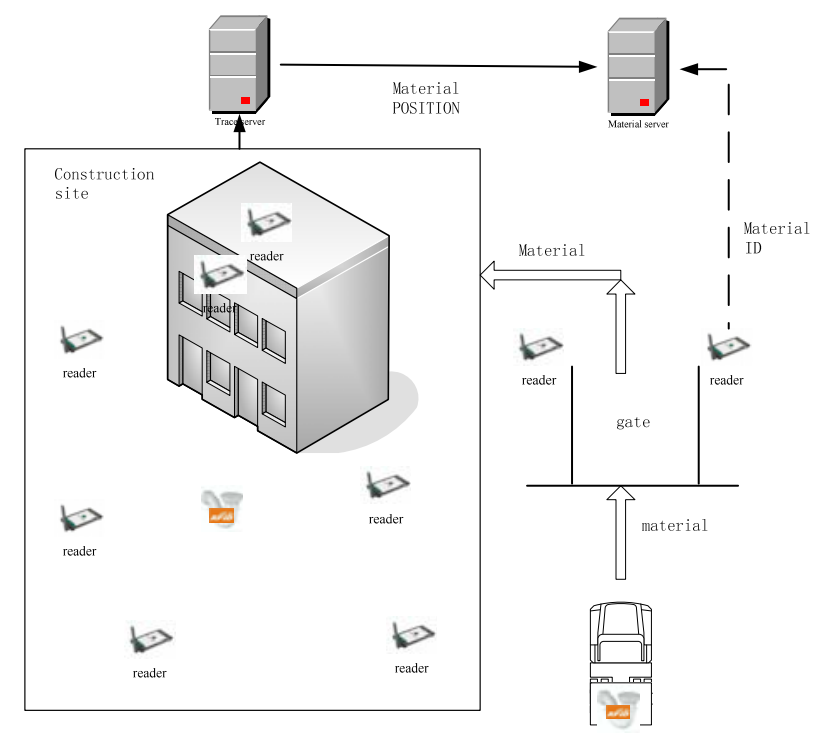

Figure 5. using 3D-PMWSN in construction project

Step 1 Attach RFID tags to the construction materials or components.

The readers installed to the entrance gate read the passive RFID labels attached to the objects (materials or components), the information of ID, size, weight, and amount and so on will be transmitted and stored in material server, which stores basic information's of all materials transported in the construction site. In the past, trucks passed directly to the construction site. By using RFID, the products' information are gathered at the same time when such products are shipped through the port.

Step 2 Install readers at the entrance of construction site to identify the materials and components.

Jongchul Song etc. have done the field tests to test the feasibility to identify the materials at the port of field site $^{[9]}$. The tests were conducted under presumed shipping pipe spools(selected construction materials) to a construction site with varying conditions: (1) the density of tags on the trailer, (2) the amount of tag data to be captured - ID only versus ID with additional data stated earlier, (3) the movement of trailer under the portal - pass through or stop-and-go at different speeds, and (4) the number of readers activated - all of the four, those two on top or side, or only one on top center of the portal. The fixed RFID system was tested in twenty-five truck passes under the portal gate, including ten passes involving reading identification and other data associated with individual pipe spools. 
The results of field tests indicated that it is technically feasible to use commercially available active RFID technology in automating the tracking of the shipping and receiving of fabricated pipe spools beyond simple identification, in typical transport conditions. In the field trials, ID and other information about pipe spools were captured from more tags when the trailer stopped for a short time under the portal, allowing the readers more time, if on the order of a few seconds to read data.

Step 3 .Scatter readers at different places in the construction site, every reader is connected to trace server.

Theoretically, the number readers and position of each reader are determined by smallest resolution of object needed positioned and the density of distribution.

(1) In the space where construction project lies in, the higher density materials are stored, the more readers should be prepared.

(2)The smallest size of objects influences the reader density: the smaller it is the more readers should be prepared.

(3)The strength of RFID also influences the reader's position and Distribution density.

(4) All of above factors should be synthetically considered to the final preparation of readers.

Step 4 According to 3D-PMAHN Localization Algorithm, readers scan the space around them timely to locate the materials near them.

Step 5 The location informations obtained are stored in trace serve.

Step 6 By above step 1 to step 5,the system can trace the material positions from the time being transported in to the dynamic changes.

\section{Conclusions}

This paper studied the most popular positioning algorithms and specially introduced a 3 dimensional positioning approach---3D-PMAHN, which is proposed by our working group. The comparisons of different features are given. 3D-PMAHN has the property of lower cost and higher positioning accuracy and can works in 3 dimensional space. This property is particularly useful for the material management in large scale construction project. We will carry out more extensive site tests to assess the exact performance in the future.

\section{References}

[1] Teizer, J., Caldas C.H., and Haas, C.T. (2007). Real-Time Three-Dimensional Occupancy Grid Modeling for the Detection and Tracking of Construction Resources, ASCE Journal of Construction Engineering and Management, 133(11), pp. 880-888, Reston, Virginia.

[2] Franc, ois Caron, Saiedeh Navabzadeh Razavi, Jongchul Song, Locating sensor nodes on construction projects,Springer Science September 2006

[3] Kefei Zhang1, Ming Zhu1.Three-Dimension Indoor Positioning Algorithms Using an Integrated RFID/INS System in Multi-storey Buildings .Location Based Services and TeleCartography II,2008:373-386

[4] Jang W S, Skibniewski M J. Wireless network-based tracking and monitoring on project sites of construction materials. In: Proceedings of the 9th International Conference on Modern Building Materials, Structures and Techniques. Vilnius, Lithuania, 2007.

[5] Hightower, J. and Borriello, G.. Location Sensing Techniques.Technical Report, Computer Science and Engineering, University of Washington,2001.

[6]http://www.aero.org/education/primers/gps/howgpsw orks.html

[7] Slobodan N. Simic and Shankar Sastry. Distributed Localization in Wireless Ad Hoc Networks, Technical Report UCB/ERL M02/26, Department of Electrical Engineering and Computer Sciences, University of California, Berkeley, CA,2002.1.

[8] NIE Wen-hui ,JU Shi-guang.A 3D-based Positioning Model and algorithm in Ad Hoc Networks,IEEE International Symposium on Education and Computer Science(2009)

[9] Jongchul Song. Tracking the location of materials on construction projects [pHD], The University of Texas at Austin,2005.

[10] SHEN Xuesong, TSINGHUA SCIENCE AND TECHNOLOGY, Wireless Sensor Networks for Resources Tracking at Building Construction Site, Volume 13, Number S1, October 2008:78-83 
NIE Wen-hui is a associate professor in the School of Computer Science and Telecommunications Engineering, Jiangsu University, Zhenjiang, PR.

China. He completed his master degrees in computerl engineering in 2008 at Jiangsu University. His research interests include MIS, software structure,embedded system, object detection, identification,location sensing and tracking.

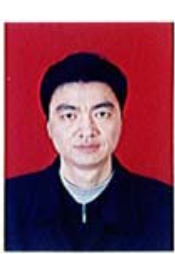

ZOU Zhi-wen is a associate professor in the School of Computer Science and Telecommunications Engineering, Jiangsu University, Zhenjiang, PR. China. He received his M.Sc in computer application technology from Jiangsu University. His research interests include spatial database, virtual reality and information security

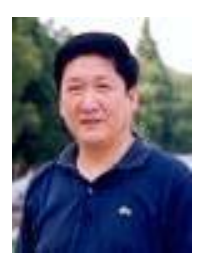

JU Shi-guang is a professor in the School of Computer Science and Telecommunications Engineering, Jiangsu University, Zhenjiang, PR. China. He received his M.Sc in computer science from Nanjing University of science and technology in 1988 and PH.D in computer science from Mexico University of Science and Technology in 1996. His research interests include spatial database and information security. DR. Ju is currently the director of the School of Computer Science and Telecommunications Engineering at Jiangsu University 